\title{
Zur Veröffentlichung der Kooperationsvereinbarung nach § 3 Absatz 2 Transfusionsgesetz
}

\author{
Friedger von Auer \\ Referat 121 "Blut und Blutprodukte, Sera und Impfstoffe, Gewebe», Bundesministerium für Gesundheit, Bonn, Germany
}

Nach $\S 1$ des Transfusionsgesetzes (TFG) ist es Zweck des Gesetzes, nicht nur für die sichere, sondern auch für die gesicherte Versorgung der Bevölkerung mit Blutprodukten zu sorgen. Diesem Gesetzeszweck entsprechend ist in $\S 3 \mathrm{Absatz}$ 1 TFG zunächst der allgemeine öffentlich-rechtliche Auftrag an die Spendeeinrichtungen geregelt, Blut und Blutbestandteile zur Versorgung der Bevölkerung mit Blutprodukten zu gewinnen. Dieser Auftrag richtet sich an die bestehenden und künftig hinzukommenden Spendeeinrichtungen im vorhandenen differenzierten System des Blutspendewesens, also an die Blutspendedienste des Deutschen Roten Kreuzes, die staatlichen und kommunalen Bluttransfusionsdienste in den Ländern, Städten und Gemeinden, an einzelne private Blutspendedienste, an die Blutspendedienste der Bundeswehr sowie an die Plasmapheresezentren der Plasma fraktionierenden Industrie.

In Anbetracht der verschiedenen Träger der Blut- und Plasmaspendeeinrichtungen hat der Gesetzgeber sodann in $\S$ 3 Absatz 2 TFG bestimmt, dass die Spendeeinrichtungen zur Erfüllung des Versorgungsauftrages zusammenarbeiten und sich gegenseitig unterstützen sollen, insbesondere im Falle des Auftretens eines Versorgungsengpasses. Dahinter steht der klare Wille des Gesetzgebers, dass kein Patient, der mit Blutprodukten behandelt werden muss, unversorgt bleibt, wenn grundsätzlich genügend Produkte bei den verschiedenen Anbietern vorhanden sind. Um dies in dem kompetitiv ausgerichteten Blutspendewesen sicher zu stellen, hat der Gesetzgeber in $\S 3$ Absatz 2 Satz 2 TFG bestimmt, dass die Spendeeinrichtungen die Einzelheiten der Zusammenarbeit in einer Vereinbarung festlegen.

Dieser Verpflichtung sind die Träger der Spendeeinrichtungen mit der Vereinbarung zu § 3 Absatz 2 TFG vom 29.
Mai 2008 [1] nachgekommen. In einem kooperativen Geist ist es gelungen, konstruktive Festlegungen zur Zusammenarbeit zu vereinbaren. Das ist ein gutes Zeichen für die Funktionsfähigkeit des deutschen Blutspendewesens, das nicht zuletzt aufgrund der Fähigkeit seiner Protagonisten zur Kommunikation und Kooperation zu einem der besten in Europa und in der Welt gerechnet werden kann.

Die Vereinbarung äußert sich zu allen relevanten Themen einer geordneten Kooperation im Blutspendewesen. Dabei geht es zunächst um die Versorgung mit Blutkomponenten im Regelfall. Sodann folgen Festlegungen zur Versorgung bei Versorgungsengpässen und in Notfallsituationen. Auch wird das Thema Werbung und Blutspendeaktionen angesprochen, um eine sachliche Werbung und einen fairen Umgang miteinander bei der Werbung von Spendern zu gewährleisten. Eine Beratungskommission soll schlichten, wenn Streitfälle zwischen Blutspendeeinrichtungen auftreten. Die Vereinbarung wird von allen relevanten Trägern von Spendeeinrichtungen und weiteren maßgeblichen Stellen und Fachkreisen mitgetragen. Das wird am Schluss der Vereinbarung durch Unterschriften oder durch getrennte Schreiben dokumentiert.

Nun muss die Vereinbarung mit Leben erfüllt werden, damit sie erfolgreich zu einer gesicherten Versorgung mit Blutprodukten beitragen kann.

\section{Literatur}

1 Bux J, Frenzel H, Seifried E, Stähle M, Müller T, Waßmann R, Rüstig W, Weinauer F, Götz G, Huck A, Stangenberg W, Kreuzer H, Hitzler W, Hutschenreuter G, Krause K-P, Scholz K, Heim M, Seidel K, Brod M, Kiefel V, Hennecke H-G: Rahmen für eine Vereinbarung nach $\$ 3$ Absatz 2 Satz 3 des Transfusionsgesetzes (TFG) zwischen den Spendeeinrichtungen in der Bundesrepublik Deutschland. Transfus Med Hemother 2010;37(4):203-208.

\section{KARGER}

Fax +497614520714

Information@Karger.de

www.karger.com
(C) 2010 S. Karger GmbH, Freiburg
Ministerialrat Friedger von Auer

Referat 121 «Blut und Blutprodukte, Sera und Impfstoffe, Gewebe»

Bundesministerium für Gesundheit

Heilsbachstraße 18, 53123 Bonn, Germany

Tel. +49 228 941-1150, Fax -4927

friedgervonauer@bmg.bund.de 